\title{
Semi-supervised Machine Learning Aided Anomaly Detection Method in Cellular Networks
}

\author{
Yutao Lu, Juan Wang, Miao Liu, Member, IEEE, Kaixuan Zhang, Tomoaki Ohtsuki, Senior Member, IEEE, \\ Guan Gui, Senior Member, IEEE, and Fumiyuki Adachi, Life Fellow, IEEE
}

\begin{abstract}
The ever-increasing amount of data in cellular networks poses challenges for network operators to monitor the quality of experience (QoE). Traditional key quality indicators (KQIs)-based hard decision methods are difficult to undertake the task of QoE anomaly detection in the case of big data. To solve this problem, in this paper, we propose a KQIs-based QoE anomaly detection framework using semi-supervised machine learning algorithm, i.e., iterative positive sample aided one-class support vector machine (IPS-OCSVM). There are four steps for realizing the proposed method while the key step is combining machine learning with the network operator's expert knowledge using OCSVM. Our proposed IPS-OCSVM framework realizes QoE anomaly detection through soft decision and can easily fine-tune the anomaly detection ability on demand. Moreover, we prove that the fluctuation of KQIs thresholds based on expert knowledge has a limited impact on the result of anomaly detection. Finally, experiment results are given to confirm the proposed IPS-OCSVM framework for QoE anomaly detection in cellular networks.
\end{abstract}

Index Terms-Machine learning, quality of experience, key quality index, one-class support vector machine, anomaly detection.

\section{INTRODUCTION}

Fifth generation $(5 \mathrm{G})$ wireless communication has three typical applications, i.e., enhanced mobile broadband (eM$\mathrm{BB}$ ), ultra reliable low latency communications (URLLC), and massive machine type communications (mMTC) [1]. According to the prediction of Cisco white paper, 5G will provide enterprisers and consumers with a large number of new applications, including anytime and anywhere video, real-time communication, super reliable communication, highdensity and large bandwidth access, high-speed mobile access, augmented reality (AR), virtual reality (VR), super large scale Internet of things [2]-[11]. However, the advent of the $5 \mathrm{G}$ era

Manuscript received month date, year; revised month date, year; month date, year. This work was supported in part by the Project Funded by the National Science and Technology Major Project of the Ministry of Science and Technology of China under Grant TC190A3WZ-2, in part by the Jiangsu Specially Appointed Professor under Grant RK002STP16001, Innovation and Entrepreneurship of Jiangsu High-level Talent under Grant CZ0010617002, in part by Summit of the Six Top Talents Program of Jiangsu under Grant XYDXX-010, in part by 1311 Talent Plan of Nanjing University of Posts and Telecommunications. (Corresponding authors: Miao Liu and Guan Gui.)

The authors are with College of Telecommunications and Information Engineering, Nanjing University of Posts and Telecommunications, Nanjing 210003, China. (e-mails: \{1019010403, 1219012920, liumiao, 1218012502, guiguan\}@njupt.edu.cn)

T. Ohtsuki is with the Department of Information and Computer Science, Keio University, Yokohama 223-8521, Japan (e-mail: ohtsuki@ics.keio.ac.jp)

F. Adachi is with the Research Organization of Electrical Communication (ROEC) Tohoku University, Sendai 980-8577 Japan (e-mail: adachi@ecei.tohoku.ac.jp) has brought about an explosive growth in wireless data traffic, while also placing higher demands on network operators to ensure users' experience.

The users' experience on the services provided by the operator is defined as quality of experience (QoE) [12], [13]. Since QoE is user-centered and subjective, it is difficult to perform accurate measurement. As an alternative way, measurable key quality indicators (KQIs) are designed for estimating QoE. KQIs can be quantified and are service based. The QoE of a service can be defined by several related KQIs for that service. Therefore, if QoE of a service is abnormal, it will be reflected in the corresponding KQIs. Big data on mobile networks brings opportunities and challenges to network optimization. At this stage, using KQIs data to monitor QoE is a common method used by the network operator. Although massive amounts of data make network optimization work difficult, we can use data-driven anomaly detection on this issue. Our research is based on KQIs data to realize anomaly detection of the corresponding QoE. Once abnormal QoE is detected, network operator can optimize the wireless network in time to achieve optimization before complaint. Undoubtedly, it is uneconomical and difficult to focus on each user's QoE. We are more concerned about the average QoE of users accessing the same cell. For example, a simple idea is to calculate the mean KQIs of users accessing the same cell to represent the cell's KQIs, and then use the cell's KQIs to determine the cell's QoE.

Traditional QoE anomaly detection method makes hard decision through thresholds and certain rules [14]. This will bring about a series of problems, which will be explained in Section II. Nowadays, both machine learning and deep learning are widely used in wireless communications [15][26] and internet of things (IoT) [27]-[31]. Also a lot of related work have done for anomaly detection in cellular networks. For example, isolation forest (IF) [32] and local outlier factor (LOF) [33] are commonly used as unsupervised methods, and stacked denoising autoencoders (SDAE) [34] and oneclass support vector machine (OCSVM) [35] are commonly used as semi-supervised methods for anomaly detection. It is worth mentioning that OCSVM is one of the most popular machine learning methods for anomaly detection since the algorithm uses only positive samples to train the classifier. Many literatures have shown that OCSVM has good applicability to various anomaly detection problems (such as intrusion detection [36], industrial monitoring [37], and data diagnosis in wireless sensor networks (WSNs) [38], [39]).

In this paper, we propose an iterative positive sample 


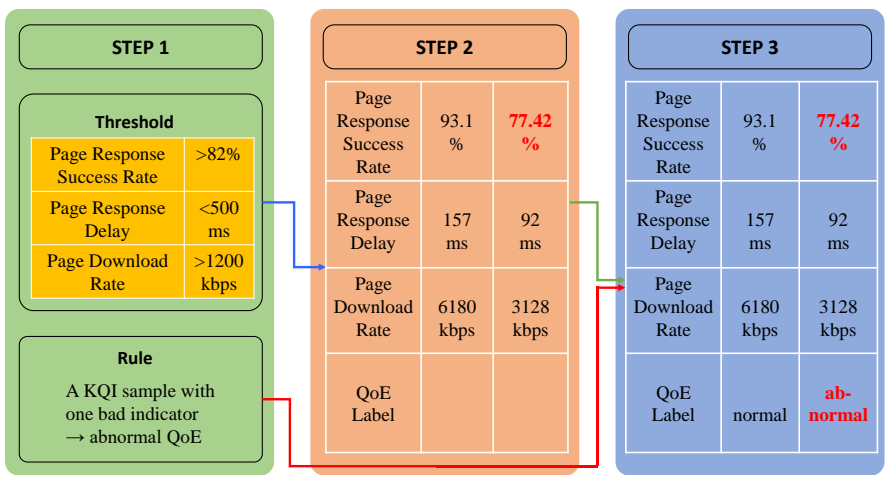

Fig. 1: Traditional KQIs-based hard decision.

aided OCSVM (IPS-OCSVM) framework to realize real-time QoE anomaly detection. Our main contributions include the following:

i) Based on KQIs data, our proposed IPS-OCSVM framework can realize real-time QoE anomaly detection through soft decision.

ii) We take full advantage of the network operator's expert knowledge on KQIs thresholds, and prove our framework is robust and can withstand slight fluctuations in KQIs thresholds.

iii) The proposed IPS-OCSVM framework can adjust the ability of anomaly detection under specific requirements, and it is very convenient for adjustment.

The remainder of this paper is organized as follows. In Section II, we discuss related work about QoE anomaly detection in cellular networks. Section III gives the problem formulation. Section IV proposes the IPS-OCSVM framework. In Section V, we analyze the performance of IPS-OCSVM. Finally, we conclude our paper in Section VI.

\section{RELATED WORK}

QoE anomaly detection in cellular networks has long been one of the most concerned issues for network operators. Most of the research on this issue focuses on studying the impact of network performance on $\mathrm{QoE}$ and the end-to-end experience as a whole. Here we first discuss the traditional KQIs-based hard decision, which is also the method that the network operator often uses. There are three main steps in traditional method: 1) Sets KQIs thresholds and makes rules; 2) Screens out bad indicators for KQIs samples, and 3) Detects KQIs samples leading to bad QoE according to the rules. For the convenience of description, we call the value of an indicator of a KQIs sample does not meet the threshold requirement as a bad indicator. Fig. 1 gives a simple example of deciding whether the QoE of web browsing service is abnormal.

The traditional KQIs-based hard decision making is very simple and convenient, however, there are often problems in actual use. Firstly, KQIs are not of the same importance. It is inaccurate to judge QoE as abnormal by the number of bad indicators. In additional, KQIs thresholds are based on network operators' expert knowledge which reflect the statistical KQIs situation of most cells, and it is difficult to adjust anomaly detection ability under different scenarios and different requirements. For example, user-intensive areas are often more difficult to secure QoE, where more radio resources are need to be allocated, so our anomaly detection methods need to be more sensitive to $\mathrm{QoE}$ anomalies in these areas [13], which is a challenging task for traditional KQIs-based hard decision method.

In [40], a three-layer mapping model from KQIs to QoE and a KQIs five-level scoring system are established for data services. Compared with the traditional hard decision method, the three-layer mapping model can better reflect the KQIs and QoE situation. The five-level scoring method provides a more detailed qualitative description of the KQIs performance. However, the traffic proportion of sub-services is time-varying, and it is not very reasonable to determine the weights of sub-services through the traffic proportion. Besides, as the complexity of the network increases, it is challenging to manually determine the exact thresholds for different KQIs in different scenarios. When the network operator finds that the result of the anomaly detection is slightly different from the actual situation and needs to fine-tune the ability of anomaly detection, then multiple thresholds need to be adjusted. In [14], an object-oriented detection framework with a two-step clustering, named as Hourglass Clustering, is used to provide anomaly codebook for QoE anomaly detection. Hourglass Clustering is a two-step clustering with dimension reducing and dimension expanding, which uses feature matching to achieve anomaly detection. Hourglass Clustering can capture the characteristics of anomalous samples, but it is also difficult to adjust the ability of QoE anomaly detection as needed.

Based on key performance indicators (KPIs) data, Peter et al. [41], Szabo1cs et al. [42], and Gabriela et al. [43] proposed KPIs-based QoE anomaly detection and diagnosis frameworks for network operators. KPIs are the measure of network performance. KPIs-based method is more convenient for network optimization, however, KPIs cannot represent QoE all the time [44]. QoE is not well estimated using KPIs data.

\section{PRoblem Formulation}

When users use the network services provided by the network operator, a large amount of KQIs data about the services will be recorded. For network operators, it does not make sense to focus on the QoE of a particular user. Network operators are more concerned with the QoE of a cluster of users, which makes network optimization more efficient and economical. Thus, we calculate the average KQIs of all users accessing a cell, and use this value to represent KQIs of the cell to estimate the cell's QoE. We use real $4 \mathrm{G}$ data from a certain network operator, which recorded the synthesized data service KQIs. Data service types include streaming services, gaming services, instant messaging services, and web browsing services. The data has a total of 41838 valid KQIs samples from 324 different cells with 14 indicators and a time span of 7 days. The most important point is that we have reference threshold for each KQI, but we do not know if QoE of each KQI sample is good or bad. Fig. 2 illustrates a scenario for estimating QoE with KQIs data. 


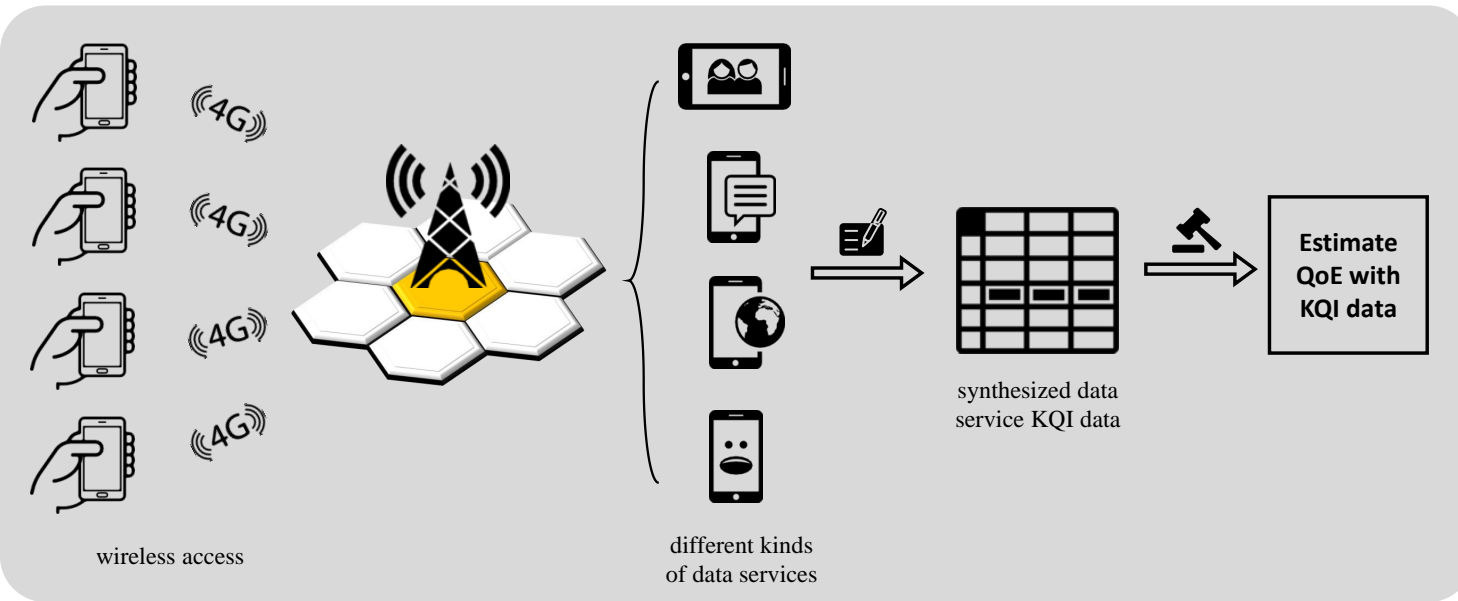

Fig. 2: Scenario description of QoE estimation using KQIs.

The data set we get has two features: large volume and high dimension. Problems are posed as follows:

- How to identify KQIs samples leading to abnormal QoE from massive data?

- How to fine-tune the ability of QoE anomaly detection in an easy way for different scenarios and different requirements?

- How to use KQIs thresholds based on expert knowledge to guide QoE anomaly detection?

\section{OUR PROPOSED IPS-OCSVM FRAMEWORK FOR KQIS-BASED QOE ANOMALY DETECTION}

In this section, we will describe the entire steps of QoE anomaly detection using IPS-OCSVM, followed by data preprocessing, learning algorithm in IPS-OCSVM, fine-tuning the parameters as needed, and implementation from offline training to online detection.

\section{A. Data Preprocessing with Expert Knowledge}

Fig. 3 shows the process of data preprocessing using KQIs thresholds based on expert knowledge. In order to take advantage of the network operator's experience in QoE anomaly detection, we draw on the network operator's KQIs threshold.s For each KQI sample, we calculate the number of bad indicators. Then we put KQIs samples with the same number of bad indicators into the same set. In this way, we divide the original large data set into several small data sets according to the number of bad indicators in KQIs samples. Fig. 4 shows the result of data preprocessing. $D_{l}$ represents a collection of samples with $l$ bad indicators.

\section{B. Learning Algorithm for the Proposed IPS-OCSVM Frame- work}

We will first briefly introduce OCSVM as a foreshadowing of our proposed IPS-OCSVM framework. In order to transform one-class classification problems into special two-class classification problems, OCSVM projects the input space from the original dimensional space into a higher dimensional space to find a hyperplane that separates the projected examples from

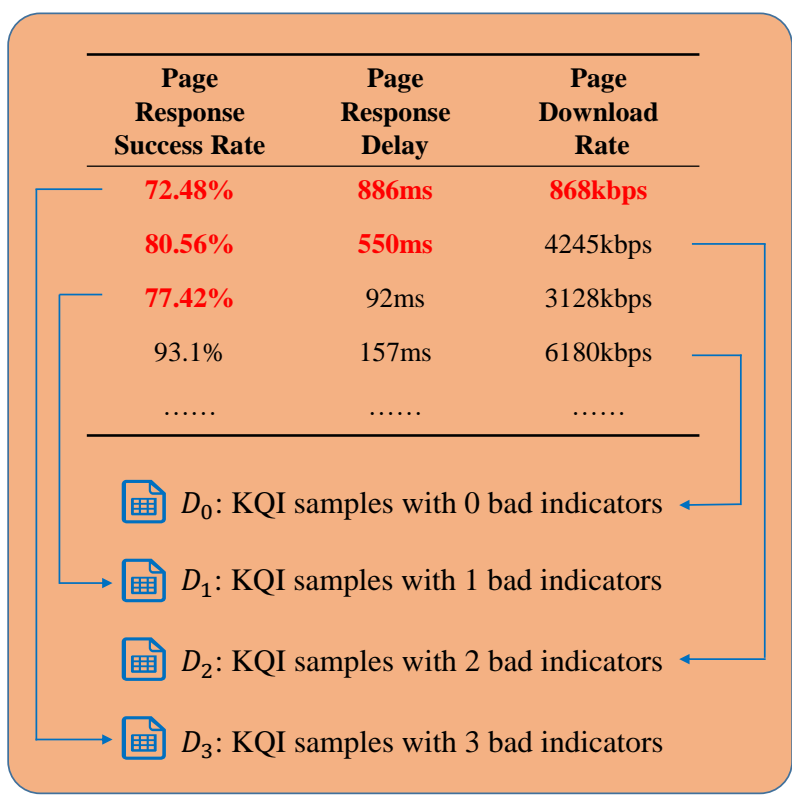

Fig. 3: Data preprocessing.

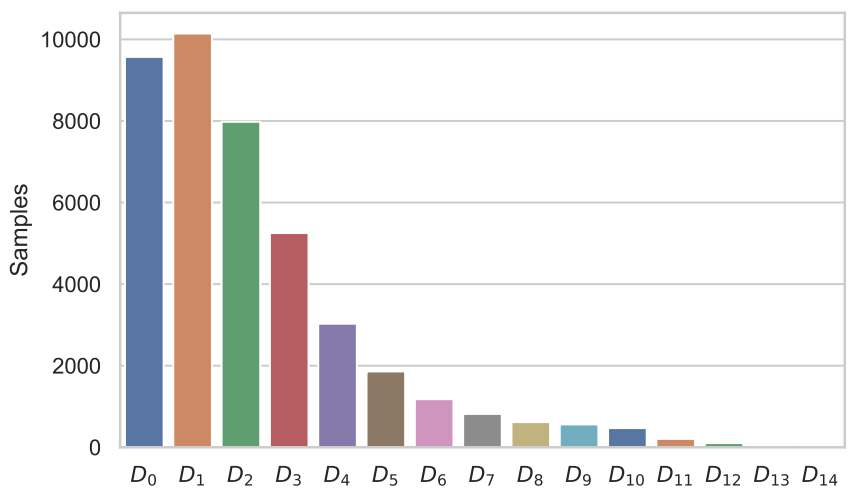

Fig. 4: KQIs samples statistical map after data preprocessing. 
the origin with the maximum possible margin. The primal quadratic problem defining the OCSVM classifier is [45]

$$
\begin{aligned}
& \mathrm{P} 1: \min _{\boldsymbol{\omega}, \xi, \rho} \frac{1}{2}\|\boldsymbol{\omega}\|^{2}+\frac{1}{\nu n} \sum_{i=1}^{n} \xi_{i}-\rho \\
& \text { s. t. } \quad \boldsymbol{\omega} \cdot \Phi\left(\boldsymbol{x}_{i}\right) \geq \rho-\xi_{i} \\
& \quad \xi_{i} \geq 0, \quad i=1,2, \ldots, n
\end{aligned}
$$

In (P1), $\boldsymbol{x}_{i}$ is the sample in the original space and $n$ is the number of training samples. $\Phi$ represents the map from the original space to the feature space, $\omega$ is the normal vector, and $\rho$ is the offset of the desired hyperplane in the feature space. The slack variable $\xi_{i}$ allows certain training samples to be misclassified. The trade-off parameter $\nu \in(0,1]$ proves to be the upper limit of the proportion of training samples that are classified outside the decision boundary, and a lower bound on the fraction of supporting vectors.

The label of $\boldsymbol{x}$ can be predicted by $f(\boldsymbol{x})$ [46], where the definition of decision function $f(\boldsymbol{x})$ is

$$
f(\boldsymbol{x})=\operatorname{sgn}(\boldsymbol{\omega} \cdot \Phi(\boldsymbol{x})-\rho)
$$

By using Lagrange multipliers, (P1) is converted to the following quadratic dual problem (P2):

$$
\begin{aligned}
\mathrm{P} 2: \quad \min _{\alpha} \frac{1}{2} \sum_{i, j} \alpha_{i} \alpha_{j} K\left(\boldsymbol{x}_{i}, \boldsymbol{x}_{j}\right) \\
\text { s. t. } \quad 0 \leq \alpha_{i} \leq 1 / \nu n \\
\quad \sum_{i=1}^{n} \alpha_{i}=1, \quad i=1,2, \ldots, n
\end{aligned}
$$

Then the decision function with a kernel expansion is given as follows:

$$
f(\boldsymbol{x})=\operatorname{sgn}\left(\sum_{i} \alpha_{i} K\left(\boldsymbol{x}_{i}, \boldsymbol{x}\right)-\rho\right)
$$

All training samples corresponding nonzero $\alpha_{i}$ are called support vectors. The kernel function $K\left(\boldsymbol{x}_{i}, \boldsymbol{x}_{j}\right)$ is designed to increase the positive sample dimension to find a hyperplane to separate them. The choice of kernel function in OCSVM is one of the key factors affecting the success of the algorithm. More details could be found in [45]. The learning algorithm in IPS-OCSVM uses OCSVM to iterate positive samples. The corresponding pseudo code is summarized in Algorithm 1.

During the initialization process, we set kernel function $K\left(\boldsymbol{x}_{i}, \boldsymbol{x}_{j}\right)=K_{0}\left(\boldsymbol{x}_{i}, \boldsymbol{x}_{j}\right)$ and $\nu=\nu_{0}$. The role of a suitable kernel function and parameter $\nu$ will be described in Section V.

Firstly, using $D_{0}$ as the original normal data to solve (P2) in OCSVM, the decision function $f(\boldsymbol{x})$ can be obtained. Secondly, the decision function is used to distinguish the normal part $N_{2}$ from the abnormal part $A_{2}$ in $D_{2}$, and the normal part $N_{2}$ in the $D_{2}$ is updated into the total normal data set $N$. By iterating according to this rule, we can get all the normal samples and the final decision function.

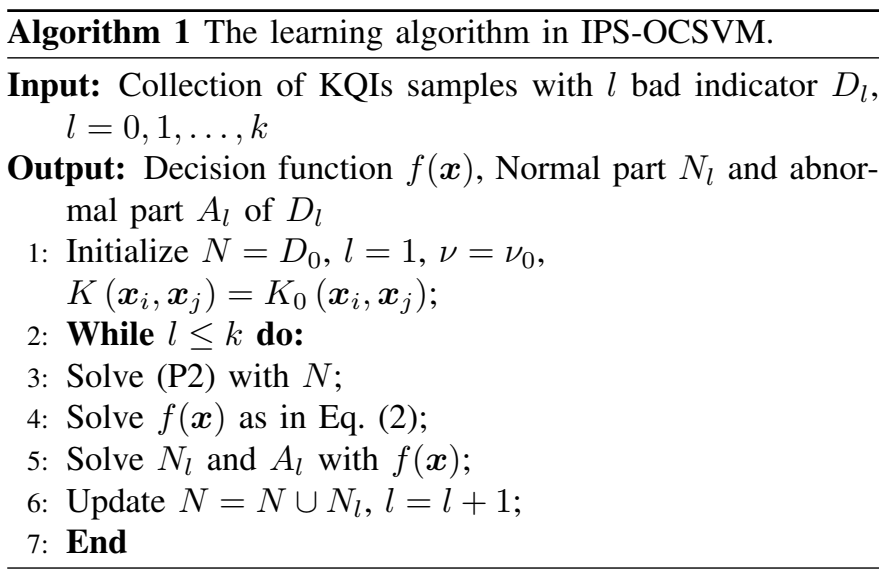

\section{Adjust the Ability of QoE Anomaly Detection}

It is one of the advantages of our method to adjust the anomaly detection ability as needed. The traditional hard decision method adjusts the QoE anomaly detection ability by adjusting the threshold. However, accurately adjusting thresholds for multiple indicators is a challenging task. Our method only needs to adjust the parameter $\nu$ to adjust the QoE anomaly detection ability based on the original threshold. Before actual use, we can compare different parameter values on a labeled data set under certain requirements to determine $\nu_{0}$. For example, the area under the receiver operating characteristic curve $(A U C)$, is widely used to estimate the predictive accuracy of classifier. Besides, recall in Eq. (9) is the fraction of the total amount of relevant instances that were actually retrieved, also used to evaluate the performance of a classifier. We record the corresponding $A U C$ and recall in different $\nu$ cases as $A(\nu)$ and $r(\nu)$. In order to obtain suitable adjustment $\nu$ for the classifier for achieving maximal $A U C$ subjects to lower bound recall, then $\nu_{0}$ can be given as

$$
\begin{aligned}
& \arg \max _{\nu} A(\nu) \\
& \text { s. t. } \\
& \quad r(\nu) \geq 0.9 \\
& 0<\nu \leq 1
\end{aligned}
$$

The impact of parameter $\nu$ on performance will be explained in the performance analysis.

\section{Offline Training and Online Detection}

After preprocessing the historical data, executing the learning algorithm in the proposed IPS-OCSVM framework and getting the decision function, we complete the whole process of offline training. Online detection can be realized by inputting real-time data into the decision function. The QoE label of a previously unseen KQIs sample can be given by $f(\boldsymbol{x})$ easily. When $f(\boldsymbol{x})=-1$, the classifier decides that this KQIs sample will result in a poor QoE. When $f(\boldsymbol{x})=1$, the classifier decides that the KQIs sample corresponds to the normal QoE. Obviously, our proposed IPS-OCSVM framework is very simple and efficient in realizing real-time QoE anomaly detection. Fig. 5 shows the flow from offline training to online detection for IPS-OCSVM framework. 


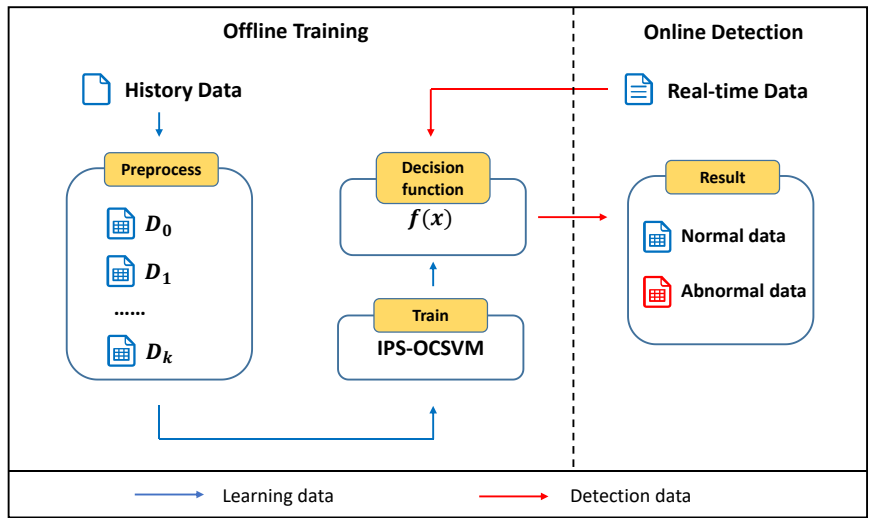

Fig. 5: From offline training to online detection for our proposed IPS-OCSVM framework.

\section{Performance Analysis}

In this section, we will analyze the IPS-OCSVM performance from the following four aspects:

- Analyze the influence of using different kernel functions on performance.

- Analyze the influence of different $\nu$ on performance.

- Analyze the influence of artificial experience fluctuation on performance.

- Evaluate performance of our proposed IPS-OCSVM framework on real test set.

\section{A. Performance of IPS-OCSVM Framework using Different Kernel Functions}

To quantitatively show that the Gaussian kernel is the best, we compare it to Linear kernel, Poly kernel, and Sigmoid kernel. The definitions of Gaussian kernel, Linear kernel, Poly kernel, and Sigmoid kernel are respectively

$$
\begin{gathered}
K\left(\boldsymbol{x}_{i}, \boldsymbol{x}_{j}\right)=\exp \left(-\frac{\left\|\boldsymbol{x}_{i}-\boldsymbol{x}_{j}\right\|^{2}}{S}\right) \\
K\left(\boldsymbol{x}_{i}, \boldsymbol{x}_{j}\right)=\boldsymbol{x}_{i}^{T} \boldsymbol{x}_{j} \\
K\left(\boldsymbol{x}_{i}, \boldsymbol{x}_{j}\right)=\left(\boldsymbol{x}_{i}^{T} \boldsymbol{x}_{j}\right)^{d} \\
K\left(\boldsymbol{x}_{i}, \boldsymbol{x}_{j}\right)=\tanh \left(\beta \boldsymbol{x}_{i}^{T} \boldsymbol{x}_{j}+\theta\right)
\end{gathered}
$$

We compare the performance of IPS-OCSVM framework using different kernel functions in Fig. 6. We observe that the curves of Linear kernel and Poly kernel are both cluttered. Linear kernel, Poly kernel and Sigmoid kernel cannot identify KQIs samples leading to bad QoE well, and obvious errors have occurred. For example, a KQIs sample with 14 bad indicators (all indicators are bad) will lead to bad QoE under normal circumstances. In other words, as the number of bad indicators increases, the final predicted abnormal proportion should converge to $100 \%$. But from the figure we can see that except for the curve of the Gaussian kernel function, the curves of the other kernel functions have some errors. Therefore in this issue we choose the Gaussian kernel function for further analysis.

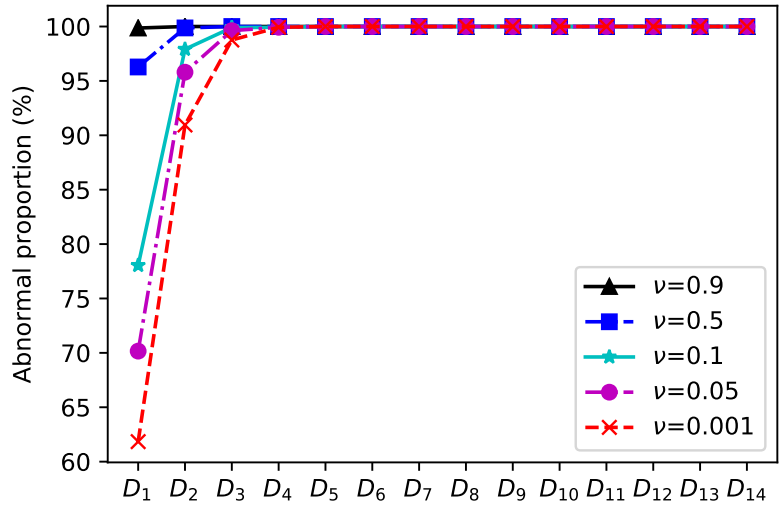

(a) Gaussian kernel

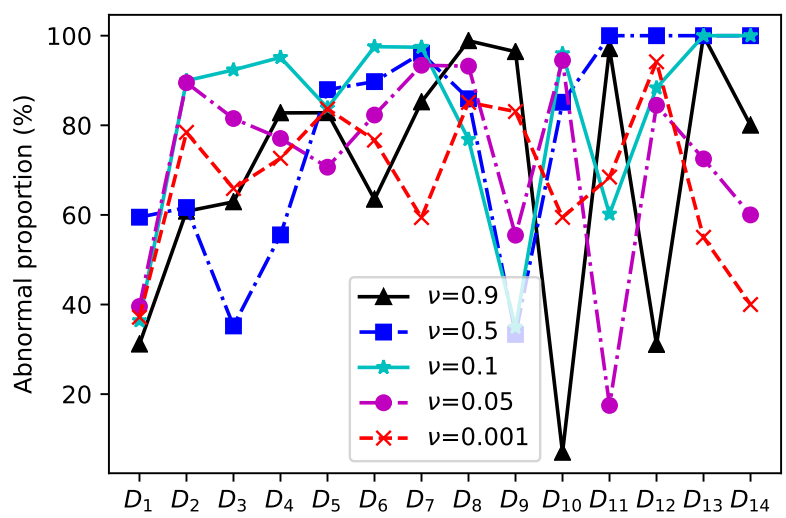

(b) Linear kernel

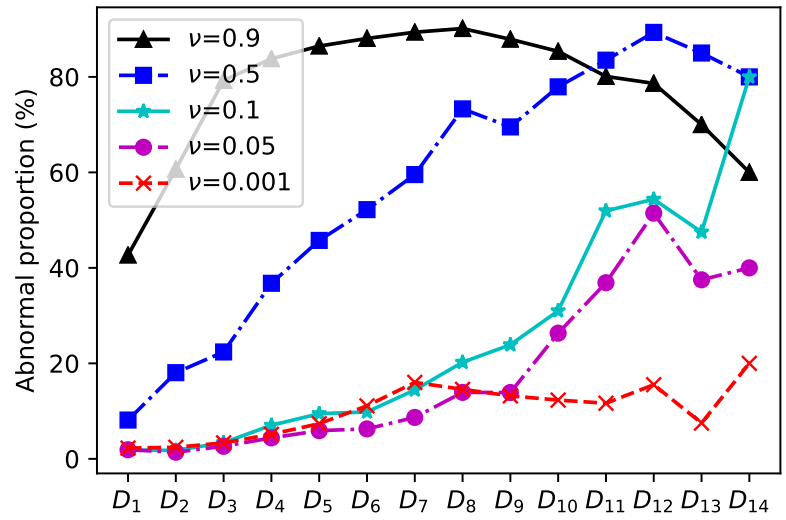

(c) Poly kernel

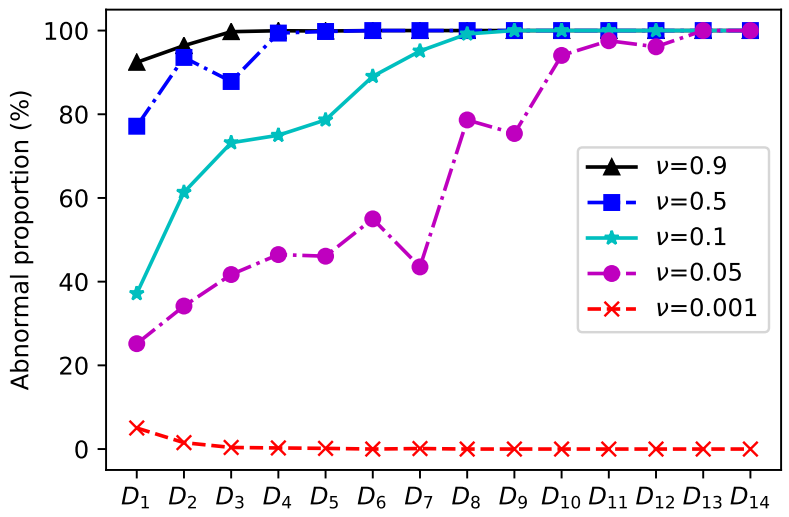

(d) Sigmoid kernel

Fig. 6: Performance comparison of the proposed IPS-OCSVM framework using different kernel functions. 


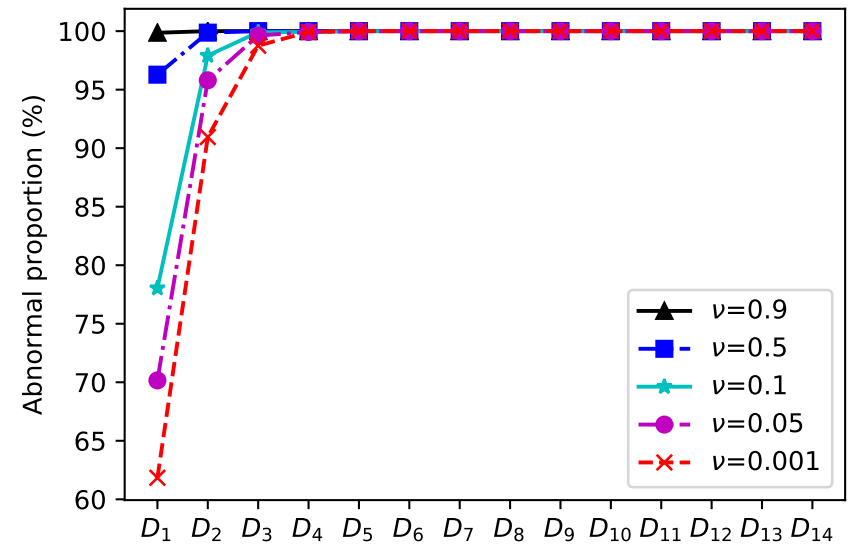

Fig. 7: Performance evaluation of IPS-OCSVM framework (Gaussian kernel) using different $\nu$.

\section{B. Performance Evaluation using Different $\nu$}

As we described in Section IV, the proposed IPS-OCSVM framework only needs to adjust the parameter $\nu$ for the adjustment of QoE anomaly detection ability. The adjustment of anomaly detection ability here means that if the network operator finds that the existing QoE anomaly detection result does not meet the actual needs, the request for determining the abnormal QoE can be tightened or relaxed. The range of parameter $\nu$ here is $(0,1]$. We selected five different $\nu$ values $(\nu \in\{0.9,0.5,0.1,0.05,0.001\})$ to study the impact of different $\nu$ on making decision.

With increasing the parameter $\nu$ in (P2), the number of support vectors and the number of misclassified training samples both increase. The parameter $\nu$ is closely related to the proportion of training samples that are misclassified outside the decision boundary. In the KQIs-based QoE anomaly detection problem, increasing the parameter $\nu$ makes the classifier more sensitive to KQIs samples with some bad indicators. A KQIs sample is more likely to be classified as a sample leading to bad QoE in the case of larger $\nu$. In Fig. 7, we can see that as $\nu$ grows, the classifier is increasingly inclined to believe that KQIs samples with bad indicators will lead to bad QoE. In actual use, the network operator can fine-tune the parameter $\nu$ for special requirements.

\section{The Influence of KQIs Thresholds Fluctuation on Perfor- mance}

In actual use, different network optimization experts may have slight differences in threshold settings under the same condition. We set up experiments to verify the impact of this KQIs thresholds fluctuation on IPS-OCSVM performance. We use the vector $\boldsymbol{t} \boldsymbol{h}$ to denote original KQIs thresholds. The upper bound of the new thresholds is 1.05th, and the lower bound is $0.95 t h$, which means that $5 \%$ random fluctuation is allowed based on the original thresholds. We selected 5 different random results with obvious difference to show in Fig. 8.

As can be seen in Fig. 8, the thresholds fluctuations within $5 \%$ have limited impact on our method, and the differences are mainly concentrated in the $D_{1}$ and $D_{2}$ data sets. To

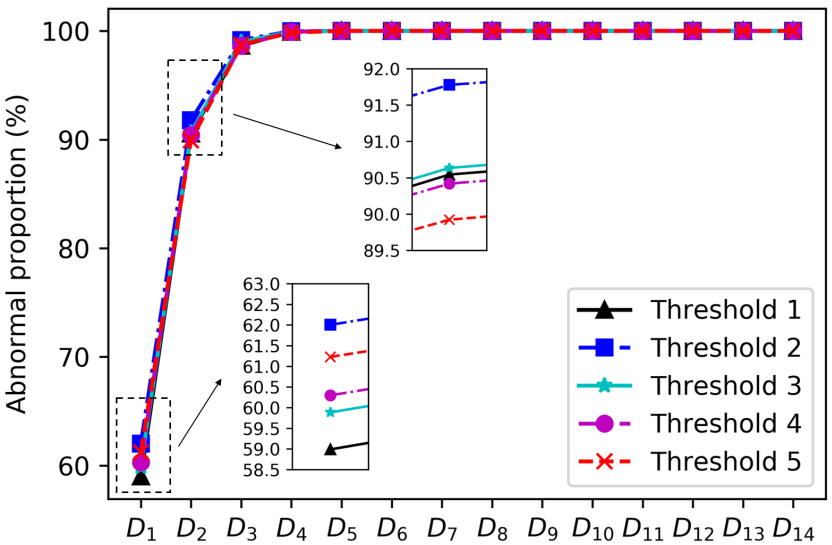

Fig. 8: Performance evaluation of the proposed IPS-OCSVM framework using different fluctuating thresholds.

investigate the effect of threshold fluctuations on the detection results, we use one-sample $t$-test, a statistical significant test, to investigate whether there was a significant difference between the multiple detection results with the fluctuating thresholds and the detection result using the original thresholds on $D_{1}$ and $D_{2}$.

Significance testing needs four steps:

i) State the null and alternative hypothesis.

ii) Calculate the test statistic.

iii) Find the $p$-value.

iv) Compare $p$-value with $\alpha$ and decide whether the null hypothesis should be rejected or accepted.

Taking the $D_{1}$ data set as an example, we test whether the detection results of the fluctuation thresholds and the detection result of the original thresholds (abnormal rate is 0.6184) are significantly different. Significance level here is $\alpha=0.05$.

Null hypothesis:

$$
H_{0}: \mu=0.6148
$$

Alternative hypothesis:

$$
H_{1}: \mu \neq 0.6148
$$

\begin{tabular}{|c|c|c|c|c|c|c|}
\hline \multicolumn{7}{|c|}{ Test Value $=0.6148$} \\
\hline & \multirow{2}{*}{$t$} & \multirow{2}{*}{ df } & \multirow{2}{*}{$\begin{array}{l}p \text {-value } \\
(2 \text {-tailed) }\end{array}$} & \multirow{2}{*}{$\begin{array}{c}\text { Mean } \\
\text { difference }\end{array}$} & \multicolumn{2}{|c|}{$\begin{array}{l}95 \% \text { confidence } \\
\text { interval of the difference }\end{array}$} \\
\hline & & & & & Lower & Upper \\
\hline$D_{1}$ & 1.069 & 20 & 0.298 & 0.0140286 & -0.013336 & 0.041393 \\
\hline
\end{tabular}

TABLE I: One-sample $t$-test on the detection results of $D_{1}$.

Table I shows the result of one-sample $t$-test [47], [48] in abnormal rate of $D_{1}$ based on IBM SPSS Statistics 21 , in which relevant parameters are listed in Table II. By comparing $p$ value $=0.298$ with significance level $\alpha=0.05$, we accept the null hypothesis, i.e., there is no significant difference between the detection results with the fluctuating thresholds and the result using the original thresholds on $D_{1}$, for $p$-value $>\alpha$. 
TABLE II: Main parameters in one-sample $t$-test.

\begin{tabular}{ll}
\hline Parameter & Description \\
\hline$t$ & $t$-statistic \\
\hline df & Degrees of freedom \\
\hline$p$-value & $\begin{array}{l}\text { Probability of observing a sample } \\
\text { statistic as extreme as the } t \text {-statistic }\end{array}$ \\
\hline Mean difference & $\begin{array}{l}\text { Difference between } \\
\text { sample mean and test value }\end{array}$ \\
\hline $\begin{array}{l}\text { 95\% confidence } \\
\text { interval of the difference }\end{array}$ & $\begin{array}{l}\text { 95\% confidence } \\
\text { interval for the population mean }\end{array}$ \\
\hline
\end{tabular}

TABLE III: One-sample $t$-test on the detection results of $D_{2}$.

\begin{tabular}{ccccccc}
\hline \multicolumn{8}{c}{ Test value $=0.9095$} \\
\cline { 5 - 7 } & \multicolumn{1}{c}{$t$} & df & $\begin{array}{c}p \text {-value } \\
(2 \text {-tailed })\end{array}$ & $\begin{array}{c}\text { Mean } \\
\text { difference }\end{array}$ & \multicolumn{2}{c}{$\begin{array}{c}\text { 95\% confidence } \\
\text { interval of the difference }\end{array}$} \\
\cline { 5 - 7 }$D_{2}$ & 1.553 & 20 & 0.136 & 0.0066238 & -0.002275 & 0.015523 \\
\hline
\end{tabular}

Table III shows the result of one-sample $t$-test in abnormal rate of $D_{2}$. Similarly, there is no significant difference between the detection results with the fluctuating thresholds and the result using the original thresholds on $D_{2}$. So far, we have shown that IPS-OCSVM can withstand slight fluctuations in the thresholds, and slight fluctuations in the thresholds have no significant effect on the final detection result.

\section{Performance Evaluation of the Proposed IPS-OCSVM Framework Based on Real Test Data}

We test the performance of IPS-OCSVM on the actual KQIs samples with QoE tags. The methods we choose to compare are semi-supervised OCSVM and SDAE (both using $D_{0}$ as training set), unsupervised IF and LOF, traditional KQIsbased hard decision as for baseline. We use precision, recall, $F 1$ and $A U C$ to evaluate the performance of the classifier. $A U C$ is the area under the $R O C$ curve, which can give an overall evaluation on the performance of different classifiers. The calculation formulas of precision, recall and $F 1$ are as follows:

$$
\begin{gathered}
\text { precision }=\frac{T P}{T P+F P} \\
\text { recall }=\frac{T P}{T P+F N} \\
F 1=\frac{2 \times \text { precision } \times \text { recall }}{\text { precision }+ \text { recall }}
\end{gathered}
$$

The classifier labels QoE of a KQIs sample as normal or abnormal (i.e. binary classification). For each predicted KQIs sample, there are only four possible outcomes, i.e. true positive (TP), true negative (TN), false positive (FP) and false negative (FN). They are respectively defined as below: TP is correctly predicted as abnormal when the instance originally labeled as abnormal. TN is correctly predicted as normal when the instance originally labeled as normal. FP is incorrectly predicted as abnormal when the instance originally labeled as normal. FN is incorrectly predicted as normal when the instance originally labeled as abnormal.

TABLE IV: Performance evaluation of the proposed IPSOCSVM framework based on the real test set which is provided by network operators.

\begin{tabular}{ccccc}
\hline Method & Precision & Recall & F1 & AUC \\
\hline IPS-OCSVM & 0.9070 & $\mathbf{0 . 9 9 0 5}$ & $\mathbf{0 . 9 4 6 9}$ & $\mathbf{0 . 8 7 4 0}$ \\
OCSVM & 0.9083 & 0.9428 & 0.9252 & 0.8578 \\
SDAE & 0.7708 & 0.9714 & 0.8596 & 0.6410 \\
IF & 0.6443 & 0.7302 & 0.6845 & 0.3840 \\
LOF & 0.6526 & 0.7397 & 0.6935 & 0.4001 \\
Baseline & $\mathbf{0 . 9 9 4 6}$ & 0.5841 & 0.736 & 0.7882 \\
\hline
\end{tabular}

Table IV shows the comparison results on the test set. By comparing the performance on the test set, we find that our proposed IPS-OCSVM framework sacrifices a little precision, but in return for a huge increase in recall, $F 1$ and $A U C$ when compared with the baseline method. The performance of OCSVM and SDAE (both using $D_{0}$ as training set) is also inferior to that of IPS-OCSVM. Moreover, the complexity and stability of SDAE are not as good as IPS-OCSVM. IF and LOF are unsupervised methods, which do not combine expert knowledge. It can be seen that their performance is far less than the performance of IPS-OCSVM, OCSVM and SDAE. IPS-OCSVM, OCSVM and SDAE all utilize expert knowledge. Besides, IF and LOF are not as convenient as IPSOCSVM in real-time detection.

\section{CONCLUSions}

In this paper, we have proposed an IPS-OCSVM framework to realize KQIs-based QoE anomaly detection in cellular networks. The proposed IPS-OCSVM can make soft decision to realize adjustment of detection ability and use expert knowledge reasonably while withstanding its fluctuation to realize fast online detection after offline training. In future work, we will consider the temporal relationship between KQIs samples, which may make anomaly detection more effective.

\section{REFERENCES}

[1] P. Popovski, K. F. Trillingsgaard, O. Simeone, and G. Durisi, "5G wireless network slicing for eMBB, URLLC, and MMTC: A communicationtheoretic view," IEEE Access, vol. 6, pp. 55 765-55 779, Sep. 2018.

[2] H. Nakayama, S. Kurosawa, A. Jamalipour, Y. Nemoto, and N. Kato, "A dynamic anomaly detection scheme for aodv-based mobile ad hoc networks," IEEE Transactions on Vehicular Technology, vol. 58, no. 5, pp. 2471-2481, Jun. 2009.

[3] Z. Zhou, X. Chen, and B. Gu, "Multi-scale dynamic allocation of licensed and unlicensed spectrum in software-defined hetnets," IEEE Network, vol. 33, no. 4, pp. 9-15, Jul./Aug. 2019.

[4] T. K. Rodrigues, K. Suto, H. Nishiyama, J. Liu, and N. Kato, "Machine learning meets computation and communication control in evolving edge and cloud: Challenges and future perspective," IEEE Communications Surveys Tutorials, to be published, doi: 10.1109/COMST.2019.2943405. 
[5] H. Zhang, B. Di, K. Bian, and L. Song, "IoT-U: Cellular internetof-things networks over unlicensed spectrum," IEEE Transactions on Wireless Communications, vol. 18, no. 5, pp. 2477-2492, May 2019.

[6] Z. Zhou, Y. Guo, Y. He, X. Zhao, and W. M. Bazzi, "Access control and resource allocation for $\mathrm{m} 2 \mathrm{~m}$ communications in industrial automation," IEEE Transactions on Industrial Informatics, vol. 15, no. 5, pp. 30933103, Mar. 2019.

[7] Z. Zhou, P. Liu, J. Feng, Y. Zhang, S. Mumtaz, and J. Rodriguez, "Computation resource allocation and task assignment optimization in vehicular fog computing: A contract-matching approach," IEEE Transactions on Vehicular Technology, vol. 68, no. 4, pp. 3113-3125, Apr. 2019.

[8] T. G. Rodrigues, K. Suto, H. Nishiyama, N. Kato, and K. Temma, "Cloudlets activation scheme for scalable mobile edge computing with transmission power control and virtual machine migration," IEEE Transactions on Computers, vol. 67, no. 9, pp. 1287-1300, Sep. 2018.

[9] T. G. Rodrigues, K. Suto, H. Nishiyama, and N. Kato, "Hybrid method for minimizing service delay in edge cloud computing through vm migration and transmission power control," IEEE Transactions on Computers, vol. 66, no. 5, pp. 810-819, May 2017.

[10] S. Zhang, H. Zhang, Q. He, K. Bian, and L. Song, "Joint trajectory and power optimization for uav relay networks," IEEE Communications Letters, vol. 22, no. 1, pp. 161-164, Jan. 2018.

[11] K. Bian, C. Gao, Y. Tao, Y. Zhang, L. Song, S. Dong, and X. Li, "Learning at the edge: Smart content delivery in real world mobile social networks," IEEE Network, vol. 33, no. 4, pp. 208-215, Jul. 2019.

[12] S. Baraković and L. Skorin-Kapov, "Survey and challenges of QoE management issues in wireless networks," Journal of Computer Networks and Communications, vol. 2013, pp. 1-10, 2013.

[13] T. Hori and T. Ohtsuki, "QoE and throughput aware radio resource allocation algorithm in LTE network with users using different applications," in IEEE Annual International Symposium on Personal, Indoor, and Mobile Radio Communications (PIMRC), Sep. 2016, pp. 1-6.

[14] X. Qin, S. Tang, X. Chen, D. Miao, and G. Wei, "SQoE KQIs anomaly detection in cellular networks: Fast online detection framework with Hourglass clustering," China Communications, vol. 15, no. 10, pp. 2537, Oct. 2018.

[15] H. Huang, Y. Peng, J. Yang, W. Xia, and G. Gui, "Fast beamforming design via deep learning," IEEE Transactions on Vehicular Technology, to be published, doi: 10.1109/TVT.2019.2949122.

[16] F. Tang, Y. Kawamoto, N. Kato, and J. Liu, "Future intelligent and secure vehicular network towards 6g: Machine learning approaches," Proceedings of the IEEE, to be published, doi: 10.1109/JPROC.2019.2954595.

[17] Y. Wang, M. Liu, J. Yang, and G. Gui, "Data-driven deep learning for automatic modulation recognition in cognitive radios," IEEE Transactions on Vehicular Technology, vol. 68, no. 4, pp. 4074-4077, Feb. 2019.

[18] H. Huang, J. Yang, H. Huang, Y. Song, and G. Gui, "Deep learning for super-resolution channel estimation and doa estimation based massive mimo system," IEEE Transactions on Vehicular Technology, vol. 67, no. 9 , pp. 8549-8560, Sep. 2018.

[19] H. Huang, S. Guo, G. Gui, Z. Yang, J. Zhang, H. Sari, and F. Adachi, "Deep learning for physical-layer $5 \mathrm{G}$ wireless techniques: Opportunities, challenges and solutions," IEEE Wireless Communications, to be published, doi: 10.1109/MWC.2019.1900027.

[20] Z. M. Fadlullah, F. Tang, B. Mao, N. Kato, O. Akashi, T. Inoue, and K. Mizutani, "State-of-the-art deep learning: Evolving machine intelligence toward tomorrow's intelligent network traffic control systems," IEEE Communications Surveys and Tutorials, vol. 19, no. 4, pp. 24322455, Apr. 2017.

[21] G. Gui, H. Huang, Y. Song, and H. Sari, "Deep learning for an effective nonorthogonal multiple access scheme," IEEE Transactions on Vehicular Technology, vol. 67, no. 9, pp. 8440-8450, Sep. 2018.

[22] H. Huang, Y. Song, J. Yang, G. Gui, and F. Adachi, "Deep-learningbased millimeter-wave massive MIMO for hybrid precoding," IEEE Transactions on Vehicular Technology, vol. 68, no. 3, pp. 3027-3032, Mar. 2019.

[23] N. Kato, Z. M. Fadlullah, B. Mao, F. Tang, O. Akashi, T. Inoue, and K. Mizutani, "The deep learning vision for heterogeneous network traffic control: Proposal, challenges, and future perspective," IEEE Wireless Communications, vol. 24, no. 3, pp. 146-153, Jun. 2017.

[24] F. Tang, B. Mao, Z. M. Fadlullah, N. Kato, O. Akashi, T. Inoue, and K. Mizutani, "On removing routing protocol from future wireless networks: A real-time deep learning approach for intelligent traffic control," IEEE Wireless Communications, vol. 25, no. 1, pp. 154-160, Feb. 2018.

[25] B. Mao, Z. M. Fadlullah, F. Tang, N. Kato, O. Akashi, T. Inoue, and K. Mizutani, "Routing or computing? the paradigm shift towards intel- ligent computer network packet transmission based on deep learning," IEEE Transactions on Computers, vol. 66, no. 11, pp. 1946-1960, Mar. 2017.

[26] Y. Zhou, Z. M. Fadlullah, B. Mao, and N. Kato, "A deep-learning-based radio resource assignment technique for $5 \mathrm{G}$ ultra dense networks," IEEE Network, vol. 32, no. 6, pp. 28-34, Nov. 2018.

[27] G. Gui, F. Liu, J. Sun, J. Yang, Z. Zhou, and D. Zhao, "Flight delay prediction based on aviation big data and machine learning," IEEE Transactions on Vehicular Technology, to be published, doi: 10.1109/TVT.2019.2954094.

[28] L. Zhao, J. Wang, J. Liu, and N. Kato, "Routing for crowd management in smart cities: A deep reinforcement learning perspective," IEEE Communications Magazine, vol. 57, no. 4, pp. 88-93, Apr. 2019.

[29] Y. Zhao, Q. Chen, W. Cao, J. Yang, J. Xiong, and G. Gui, "Deep Learning for Risk Detection and Trajectory Tracking at Construction Sites," IEEE Access, vol. 7, pp. 30905-30912, Dec. 2019.

[30] L. Shao, C. Liang, K. Wang, W. Cao, W. Zhang, G. Gui, and H. Sari, "Attention gan-based method for designing intelligent making system," IEEE Access, vol. 7, pp. 163097-163 104, Apr. 2019.

[31] J. Pan, Y. Yin, J. Xiong, W. Luo, G. Gui, and H. Sari, "Deep learningbased unmanned surveillance systems for observing water levels," IEEE Access, vol. 6, pp. 73 561-73 571, Nov. 2018.

[32] F. T. Liu, K. M. Ting, and Z.-H. Zhou, "Isolation Forest," in IEEE International Conference on Data Mining, 2008, pp. 413-422.

[33] M. M. Breunig, H.-P. Kriegel, R. T. Ng, and J. Sander, "LOF: Identifying density-based local outliers," in ACM Sigmod Record, vol. 29, no. 2, 2000, pp. 93-104.

[34] P. Vincent, H. Larochelle, I. Lajoie, Y. Bengio, and P.-A. Manzagol, "Stacked denoising autoencoders: Learning useful representations in a deep network with a local denoising criterion," Journal of Machine Learning Research, vol. 11, no. 12, pp. 3371-3408, Dec. 2010.

[35] B. Schölkopf, R. C. Williamson, A. J. Smola, J. Shawe-Taylor, and J. C. Platt, "Support vector method for novelty detection," in Advances in Neural Information Processing Systems, 2000, pp. 582-588.

[36] L. A. Maglaras, J. Jiang, and T. J. Cruz, "Combining ensemble methods and social network metrics for improving accuracy of OCSVM on intrusion detection in SCADA systems," Journal of Information Security and Applications, vol. 30, pp. 15-26, Oct. 2016.

[37] F. Harrou, A. Dairi, Y. Sun, and F. Kadri, "Detecting abnormal ozone measurements with a deep learning-based strategy," IEEE Sensors Journal, vol. 18 , no. 17 , pp. $7222-7232$, Sep. 2018.

[38] X. Miao, Y. Liu, H. Zhao, and C. Li, "Distributed Online One-Class Support Vector Machine for Anomaly Detection Over Networks," IEEE Transactions on Cybernetics, vol. 49, no. 4, pp. 1475-1488, Apr. 2019.

[39] O. Ghorbel, W. Ayedi, H. Snoussi, and M. Abid, "Fast and Efficient Outlier Detection Method in Wireless Sensor Networks," IEEE Sensors Journal, vol. 15, no. 6, pp. 3403-3411, Jun. 2015.

[40] M. Li, H. Wei, and H. Liao, "Mobile terminal quality of experience analysis based on big data," in International Symposium on Communications and Information Technologies (ISCIT), 2016, pp. 241-245.

[41] P. Szilágyi and S. Nováczki, "An automatic detection and diagnosis framework for mobile communication systems," IEEE Transactions on Network and Service Management, vol. 9, no. 2, pp. 184-197, Mar. 2012.

[42] S. Nováczki, "An improved anomaly detection and diagnosis framework for mobile network operators," in International Conference on the Design of Reliable Communication Networks, 2013, pp. 234-241.

[43] G. F. Ciocarlie, U. Lindqvist, S. Nováczki, and H. Sanneck, "Detecting anomalies in cellular networks using an ensemble method," in Proceedings of the International Conference on Network and Service Management (CNSM), 2013, pp. 171-174.

[44] G. Song, W. Wang, D. Chen, and T. Jiang, "KPI/KQI-driven coordinated multipoint in 5g: Measurements, field trials, and technical solutions," IEEE Wireless Communications, vol. 25, no. 5, pp. 23-29, October 2018.

[45] B. Schölkopf, J. C. Platt, J. Shawe-Taylor, A. J. Smola, and R. C. Williamson, "Estimating the support of a high-dimensional distribution," Neural Computation, vol. 13, no. 7, pp. 1443-1471, Jul. 2001.

[46] Z. Ghafoori, S. M. Erfani, S. Rajasegarar, J. C. Bezdek, S. Karunasekera, and C. Leckie, "Efficient unsupervised parameter estimation for oneclass support vector machines," IEEE Transactions on Neural Networks and Learning Systems, vol. 29, no. 10, pp. 5057-5070, Jan. 2018.

[47] N. Cressie, L. Sheffield, and H. Whitford, "Use of the one sample ttest in the real world," Journal of Chronic Diseases, vol. 37, no. 2, pp. 107-114, 1984

[48] J. C. De Winter, "Using the Student's t-test with extremely small sample sizes," Practical Assessment, Research \& Evaluation, vol. 18, no. 10, Aug. 2013. 


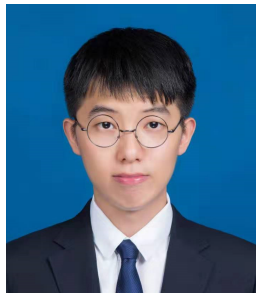

Yutao Lu (S'19) Yutao Lu received the B.Sc. Degree in communication engineering from Nanjing University of Posts and Telecommunications, Nanjing, China in 2019. He is currently pursuing the $\mathrm{PhD}$ degree in communication and information engineering with the Nanjing University of Posts and Telecommunications, Nanjing, China. Her research interest includes machine learning for wireless communications.

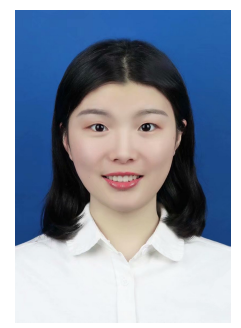

Juan Wang (S'19) Juan Wang received the B.Sc. Degree in communication engineering from Jinling Institute of Technology, Nanjing, China in 2019. She is currently pursuing the $\mathrm{PhD}$ degree in communication and information engineering with the Nanjing University of Posts and Telecommunications, Nanjing, China. She has published 8 papers in international journals and conferences. Her research interest includes machine learning for wireless communications.

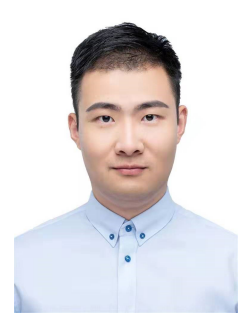

munications.
Miao Liu (M'18) received the B.Sc. Degree in communication engineering from University of Electric Science and Technology of China, Cheng China in 2011, M.Sc degree, $\mathrm{PhD}$ degree in communication engineering from Southeast University, Nanjing, China and 2013 and 2019, respectively. $\mathrm{He}$ is assistant professor with Nanjing University of Posts and Telecommunications, Nanjing China. $\mathrm{He}$ has published more than 15 papers in IEEE international journal and conferences. His research interest includes machine learning for wireless com-

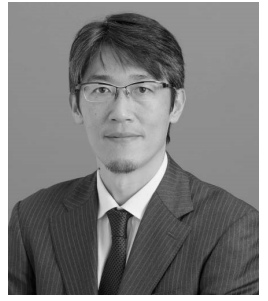

Tomoaki Ohtsuki (SM'01) received the B.E., M.E., and $\mathrm{Ph} . \mathrm{D}$. degrees in electrical engineering from Keio University, Yokohama, Japan, in 1990, 1992, and 1994, respectively. From 1994 to 1995, he was a Post-Doctoral Fellow and a Visiting Researcher in electrical engineering with Keio University. From 1993 to 1995, he was a Special Researcher of Fellowships of the Japan Society for the Promotion of Science for Japanese Junior Scientists. From 1995 to 2005 , he was with the Science University of Tokyo. In 2005, he joined Keio University, where he is currently a Professor. From 1998 to 1999 , he was with the Department of Electrical Engineering and Computer Sciences, University of California at Berkeley, Berkeley. He is engaged in research on wireless communications, optical communications, signal processing, and information theory. He was a recipient of the 1997 Inoue Research Award for Young Scientist, the 1997 Hiroshi Ando Memorial Young Engineering Award, Ericsson Young Scientist Award 2000, the 2002 Funai Information and Science Award for Young Scientist, the IEEE the 1st Asia Pacific Young Researcher Award 2001, the 5th International Communication Foundation (ICF) Research Award, the 2011 IEEE SPCE Outstanding Service Award, the 27th TELECOM System Technology Award, the ETRI Journal's 2012 Best Reviewer Award, and the Best Paper Award at the 9th International Conference on Communications and Networking in China 2014.

He has published more than 165 journal papers and 380 international conference papers. He served a Chair for the IEEE Communications Society and the Signal Processing for Communications and Electronics Technical Committee. He is a fellow of the IEICE. He was the Vice President of Communications Society of the IEICE and is the President-Elect of Communications Society of the IEICE. He served as a Technical Editor for the IEEE Wireless Communications Magazine and an Editor for the Elsevier Physical Communications. He is currently serving an Area Editor for the IEEE Transactions on Vehicular Technology and an Editor for the IEEE Communications Surveys and Tutorials. He has served as the General CoChair, the Symposium Co-Chair, and the TPC Co-Chair of many conferences, including the IEEE GLOBECOM 2008, SPC, IEEE ICC2011, CTS, IEEE GCOM2012, SPC, IEEE APWCS, and IEEE SPAWC. He gave tutorials and keynote speech at many international conferences, including the IEEE VTC and IEEE PIMRC.

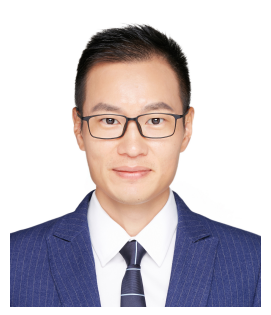

Guan Gui (M'11-SM'17) received the Dr. Eng degree in Information and Communication Engineering from University of Electronic Science and Technology of China, Chengdu, China, in 2012. From October 2009 to March 2012, with the financial supported from the China scholarship council (CSC) and the global center of education (ECOE) of Tohoku University, he joined the Department of Communications Engineering, Graduate School of Engineering, Tohoku University as for research assistant as well as postdoctoral research fellow, respectively. From September 2012 to March 2014, he was supported by Japan society for the promotion of science (JSPS) fellowship as postdoctoral research fellow at same laboratory. From April 2014 to October 2015, he was an Assistant Professor in Department of Electronics and Information System, Akita Prefectural University. Since November 2015, he has been a professor with Nanjing University of Posts and Telecommunications, Nanjing, China.

$\mathrm{He}$ is currently engaged in research of deep learning, compressive sensing and advanced wireless techniques. Dr. Gui has published more than 200 international peer-reviewed journal/conference papers. He received Member and Global Activities Contributions Award in IEEE ComSoc and eight best paper awards, i.e., ICEICT 2019, CSPS 2019, ADHIP 2018, CSPS 2018 , ICNC 2018, ICC 2017, ICC 2014 and VTC 2014-Spring. He was also selected as for Jiangsu Specially-Appointed Professor (2016), Jiangsu Highlevel Innovation and Entrepreneurial Talent (2016), Jiangsu Six Top Talent (2018), Nanjing Youth Award (2018). Dr. Gui was an Editor of Security and Communication Networks (2012-2016). He has been the Editor of IEEE Transactions on Vehicular Technology, since 2017, the Editor of Physical Communication, since 2019, the Editor of Wireless Networks, since 2019 , the Editor of IEEE Access, since 2018, the Editor of KSII Transactions on Internet and Information Systems since 2017, the Editor of Journal on Communications, since 2019, and the Editor-in-Chief of EAI Transactions on Artificial Intelligence, since 2018. He is IEEE Senior Member. 


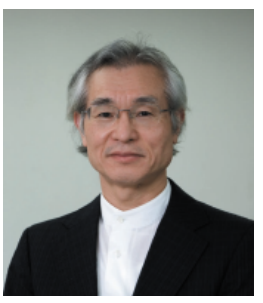

Fumiyuki Adachi (M'79-SM'90-F'02-LF'16) received the B.S. and Dr. Eng. degrees in electrical engineering from Tohoku University, Sendai, Japan, in 1973 and 1984, respectively. In April 1973, he joined NTT Laboratories and conducted various researches on digital cellular mobile communications. From July 1992 to December 1999, he was with NTT DoCoMo, where he led a research group on Wideband CDMA for 3G systems. In January 2000 he joined Tohoku University, Sendai, Japan, where he was a Professor at the Dept. of Communications Engineering, Graduate School of Engineering until he retired in March 2016. Currently, Prof. Adachi is a specially-appointed professor in the Research Organization of Electrical Communication at Tohoku University, and is continuing his research interest is in the area of wireless signal processing (multiaccess, equalization, antenna diversity, adaptive transmission, channel coding, etc.) and wireless networking. He is an IEICE Fellow and an IEEE Fellow. He is a recipient of the IEEE Vehicular Technology Society Avant Garde Award 2000, IEICE Achievement Award 2002, Thomson Scientific Research Front Award 2004, Ericsson Telecommunications Award 2008, Telecom System Technology Award 2009, Prime Minister Invention Award 2010 British Royal Academy of Engineering Distinguished Visiting Fellowship 2011, KDDI Foundation Excellent Research Award 2012, VTS Conference Chair Award 2014, C\&C Prize 2014, and IEEE VTS Stuart Meyer Memorial Award 2017, and IEEE ComSoc RCC Technical Recognition Award 2017. 